\title{
РАСШИРЕНИЕ ФУНКЦИОНАЛА ИНТЕЛЛЕКТУАЛЬНЫХ АВТОРУЛЕВЫХ ПРОГРАММНО-АППАРАТНЫМ КОМПЛЕКСОМ КОНТРОЛЯ ДВИЖЕНИЯ В ПРИБРЕЖНЫХ ВОДАХ
}

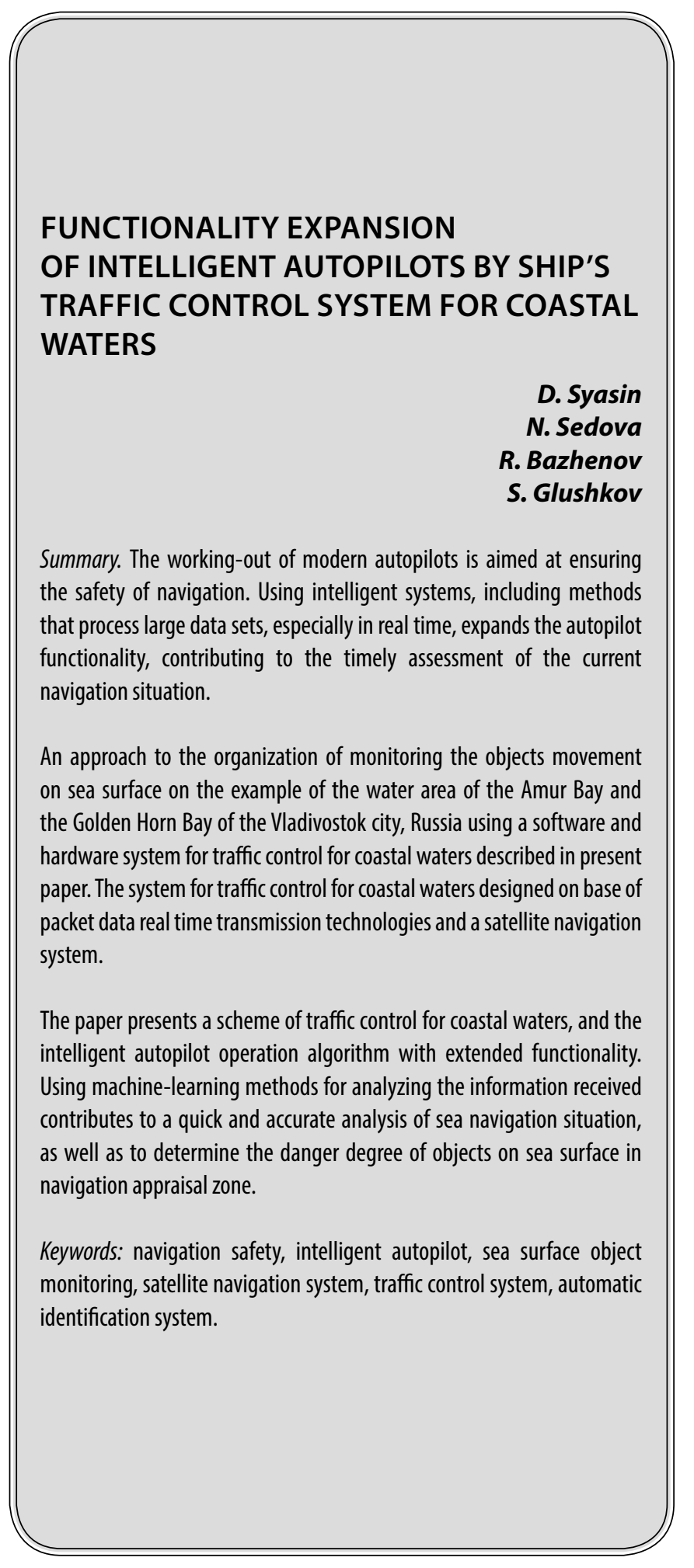

Сясин Дмитрий Юрьевич

Руководитель проектов, Московский государственный университет имени М.В. Ломоносова dims5@mail.ru

Седова Нелли Алексеевна

К.т.н., доцент, Морской государственный университет им. адм. Г.И. Невельского, г. Владивосток

nellyfish81@mail.ru

Баженов Руслан Иванович

К.n.н., доцент, Приамурский государственный университет им. Шолом-Алейхема, г. Биробиджан r-i-bazhenov@yandex.ru

Глушков Сергей Витальевич

Д.т.н., профессор, Морской государственный университет им. адм. Г.И. Невельского, г. Владивосток

glushkov@msun.ru

Аннотация. Разработка современных авторулевых направлена на обеспечение безопасности судоходства. Использование интеллектуальных систем, в том числе методов, осуществляющих обработку большого количества данных, особенно в режиме реального времени, расширяет функционал авторулевого, способствуя своевременной оценке сложившейся навигационной обстановки. Описывается подход к организации наблюдения за движением морских надводных объектов на примере акватории Амурского залива и бухты Золотой рог города Владивосток с использованием программно-аппаратного комплекса контроля движения надводных объектов в прибрежных водах на основе технологий пакетной передачи данных в режиме реального времени и спутниковой системы навигации. В работе приведена схема комплекса наблюдения за надводными объектами, а также алгоритм работы интеллектуального авторулевого с расширенным функционалом. Применение методов машинного обучения для анализа полученной информации способствует быстрому и достаточно точному анализу надводной обстановки, а также определению степени опасности объектов, попавших в зону оценки ситуации сближения.

Ключевые слова: безопасность мореплавания, интеллектуальный авторулевой, наблюдение за движением надводных объектов, спутниковая система навигации, система управления движением судов, автоматическая идентификационная система. 


\section{Ввемение}

$\Pi$ роблема обеспечения безопасности мореплавания, которая во многом зависит от технических средств, выполняющих автоматизированное наблюдение за движением судов и других надводных объектов в заданном районе, является одной из главных в прибрежных водах с активным судоходством, в частности при организации работы морских портов. В настоящее время эту задачу решают системы управления движением судов (СУДС). Они создаются, в первую очередь, в районах и портах с интенсивным судоходством и повышенной экологической опасностью. Три наиболее важных задачи, решаемые СУДС, это организация движения судов, информационное обеспечение и навигационная помощь [1]. В настоящее время СУДС задействованы в России в портах Новороссийска, Санкт-Петербурга Архангельска, Владивостока, Калининграда, Мурманска, Находки, Балтийска, Ванино, порта Восточный и др. [2].

В Приморском крае обеспечение безопасности судоходства обеспечивается СУДС залива Петра Великого [3]. СУДС залива Петра Великого функционирует на акваториях следующих морских портов: порт города Владивосток, порт города Находка, порт посёлка Зарубино и других. Одним из важнейших компонентов СУДС залива Петра Великого является сеть связи и передачи данных, основная задача которой заключается в получении информации с радиотехнических постов. Основой таких сетей связи и передачи данных до сих пор составляют радиорелейные линии связи, которые имеют ряд недостатков, которые ограничивают их функционирование. К таким недостаткам можно отнести влияние интерференции на качество передачи информации (при расположении радиорелейных каналов связи над морской поверхностью), практическую невыполнимость реализации полносвязной топологии сети связи и передачи данных, неконкурентную первоначальную стоимость передаваемой информации и нехватку согласованных межсетевых протоколов обмена информацией [4].

Одним из основных источников данных о надводных объектах в зоне действия СУДС для решения вышеперечисленных задач является автоматическая идентификационная система (АИС) - Automatic Identification System, обеспечивающая идентификацию судов и автоматизированный обмен наиболее важными идентификационными данными между судами и береговыми станциями с использованием каналов радиосвязи [5]. АИС включает в себя мобильные станции (транспондеры), устанавливаемые на судах и на других объектах, радиоканалы, береговые станции, репитеры, информационную сеть, связывающую базовые станции с береговыми службами, и оборудование, устанавливаемое в береговых службах. Сообщения, передаваемые АИС, содержат идентифика- ционные данные, данные о состоянии объекта, получаемые с элементов управления объектом, данные о географических координатах и информацию, вводимую вручную обслуживающим персоналом объекта.

Установка оборудования АИС на судах осуществляется в соответствии с Правилом V/19 Международной конвенции по охране человеческой жизни на море и связанными с ним резолюциями. Согласно этому правилу АИС оборудуются суда валовой вместимостью 300 и более, совершающие международные рейсы, и суда валовой вместимостью 500 и более, не совершающие международные рейсы. При этом АИС дополняет радиолокационную станцию и другие средства наблюдения за навигационной обстановкой, а также как средство обмена информацией с береговыми службами. В прибрежных районах активного судоходства подавляющая часть судов, в основном маломерных, не оборудована автоматической идентификационной системой, что негативно влияет на безопасность судоходства в таких районах [6].

\section{Программно-аппаратный

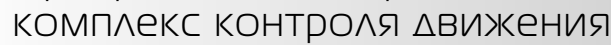 в прибрежных во $\triangle \mathrm{ax}$}

В условиях повышенной интенсивности судоходства в прибрежных водах, на подходах к портам, в узкостях существует проблема контроля движения судов, не оборудованных приёмниками АИС. К такой категории судов относятся, как правило, маломерные суда и малые моторные и парусные суда, участвующие в общем движении наряду с конвенционными морскими судами. Возможности контроля движения таких судов весьма незначительны в условиях ограниченной видимости, наличия препятствий для радиолокационного наблюдения, что создаёт дополнительные трудности в расхождении судов на акватории. В связи с этим возникает необходимость разработки технологий контроля движения таких судов, ориентированных, в первую очередь, на дешевизну и доступность их владельцам. Таким образом, актуальность исследования не вызывает сомнений.

Разрабатываемый программно-аппаратный комплекс, предназначенный для интеграции с интеллектуальным авторулевым, и позволяющим дополнить информационный ресурс СУДС данными о надводных объектах, не оснащённых АИС. Так как основная масса таких объектов - маломерные суда (парусные яхты, моторные и гребные лодки, катера), основными критериями, предъявляемыми к такому комплексу, являются стоимость реализации, простота использования и установки. Для идентификации надводных объектов в прибрежных водах в разрабатываемом подходе предлагается использовать данные глобальной системы определения координат (Global Positioning System — GPS) [7], а передачу 


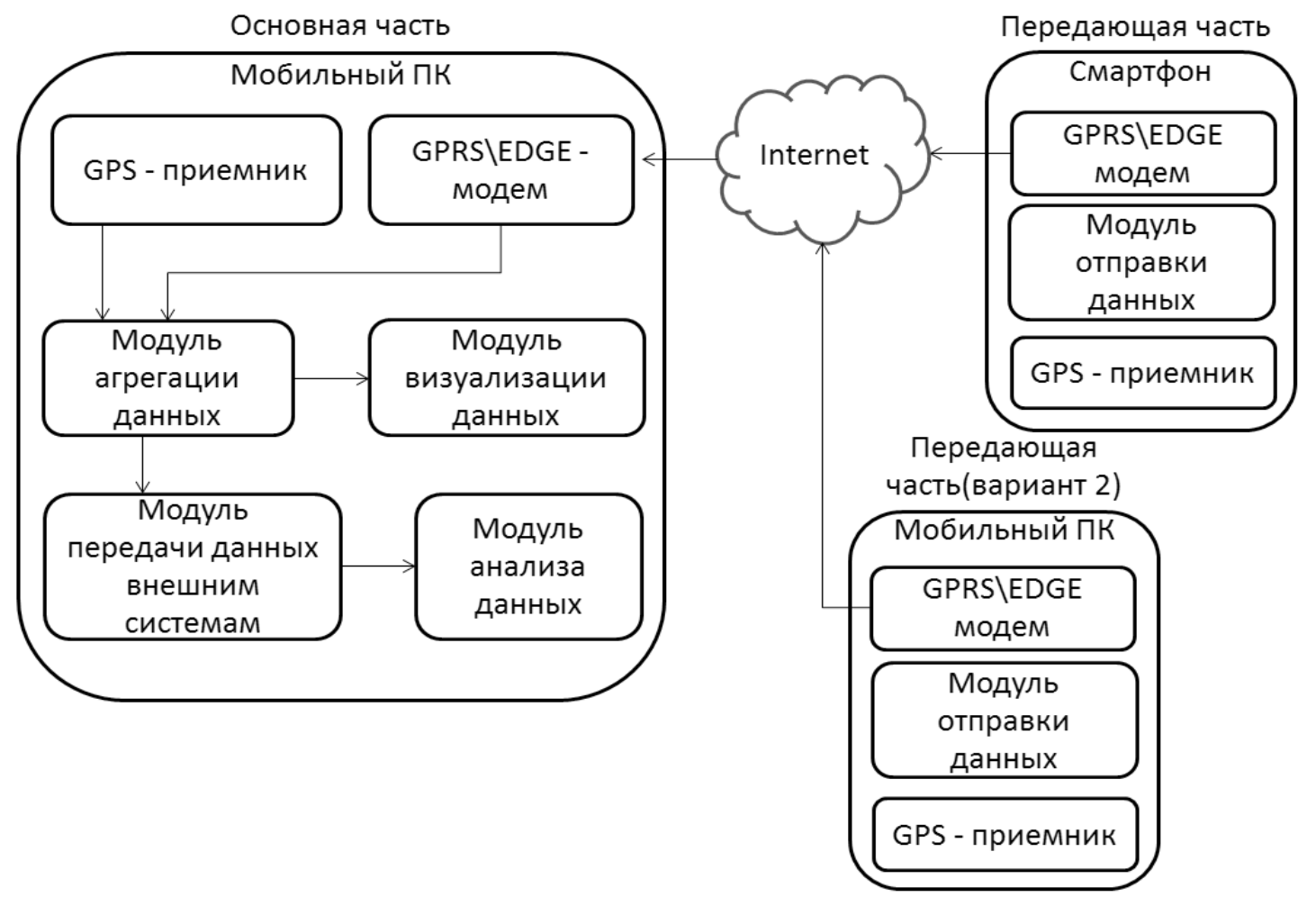

Рис. 1. Схема программно-аппаратного комплекса наблюдения за надводными объектами

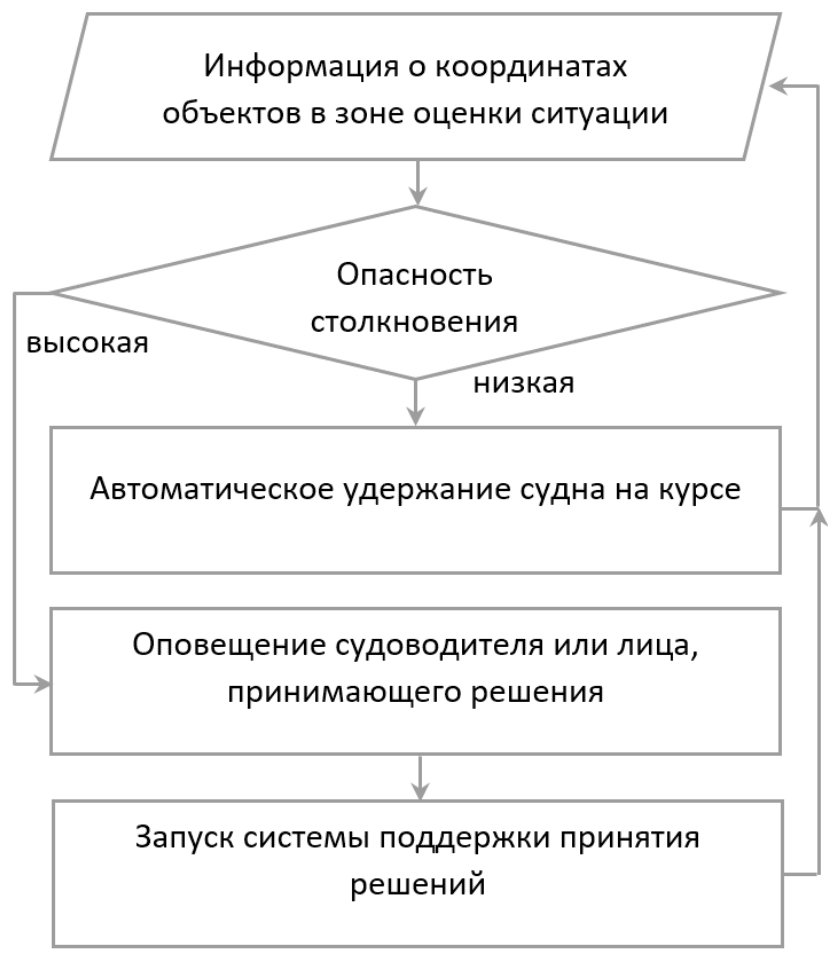

Рис. 2. Алгоритм работы интеллектуального авторулевого с расширенным функционалом

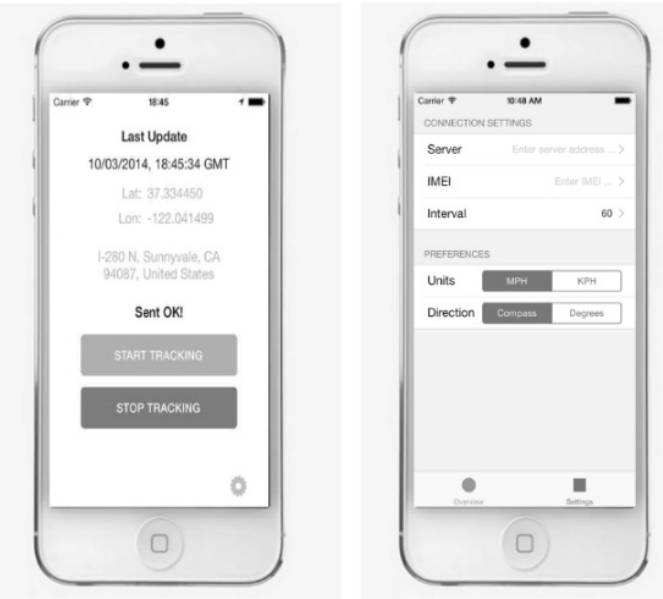

Рис. 3. Внешний вид интерфейса передающей части комплекса
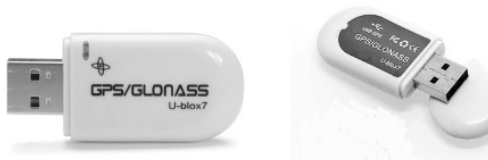

Рис. 4. Внешний вид интерфейса передающей части комплекса 


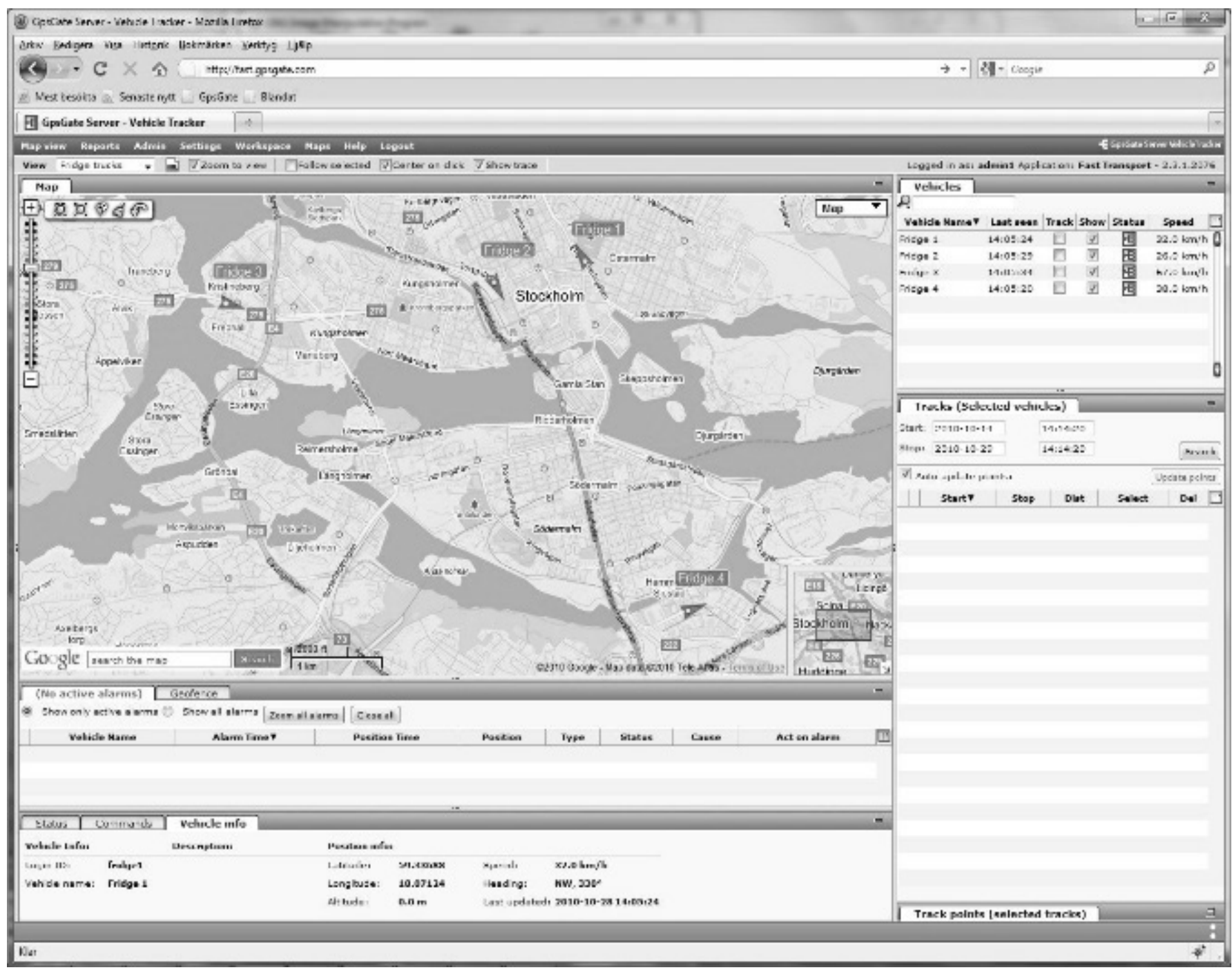

Рис. 5. Внешний вид пользовательского интерфейса Franson GPS Gate Server

данных осуществлять в реальном времени через сеть сотовой связи по протоколу пакетной радиосвязи общего пользования (General Packet Radio Service - GPRS) [8]. Таким образом, функционально предлагаемый программно-аппаратный комплекс делится на две части: передающая часть, устанавливаемая на наблюдаемом судне, и основная часть, агрегирующая и обрабатывающая полученные данные, как показано на рисунке 1. Использование простых и широко распространенных функциональных элементов комплекса - мобильных телефонов с функцией GPS и GPRS позволит получать актуальную информацию в реальном времени о движении надводных объектов в прибрежных водах, таким образом повышая информационное обеспечение безопасности судоходства.

Одним из направлений развития предложенного подхода является интеграция основной и передающей частей комплекса в структуру интеллектуального авторулевого с единым графическим интерфейсом и последующей обработкой входящей информации. Подобного рода задачи хорошо решаются с привлечением машинного обучения и позволят значительно расширить функциональные возможности авторулевого, который помимо удержания судна на курсе получит возможность идентификации потенциально аварийных ситуаций в зоне чрезмерного сближения, их обработки и выдачи рекомендаций навигатору по маневру расхождения [9]. Использование именно методов машинного обучения позволяет обеспечить быструю и достаточно точную обработку большого количества информации в режиме реального времени.

Такой авторулевой, принимая координаты надводных объектов в зоне оценки ситуации в реальном времени вычисляет скорость и направление движения 


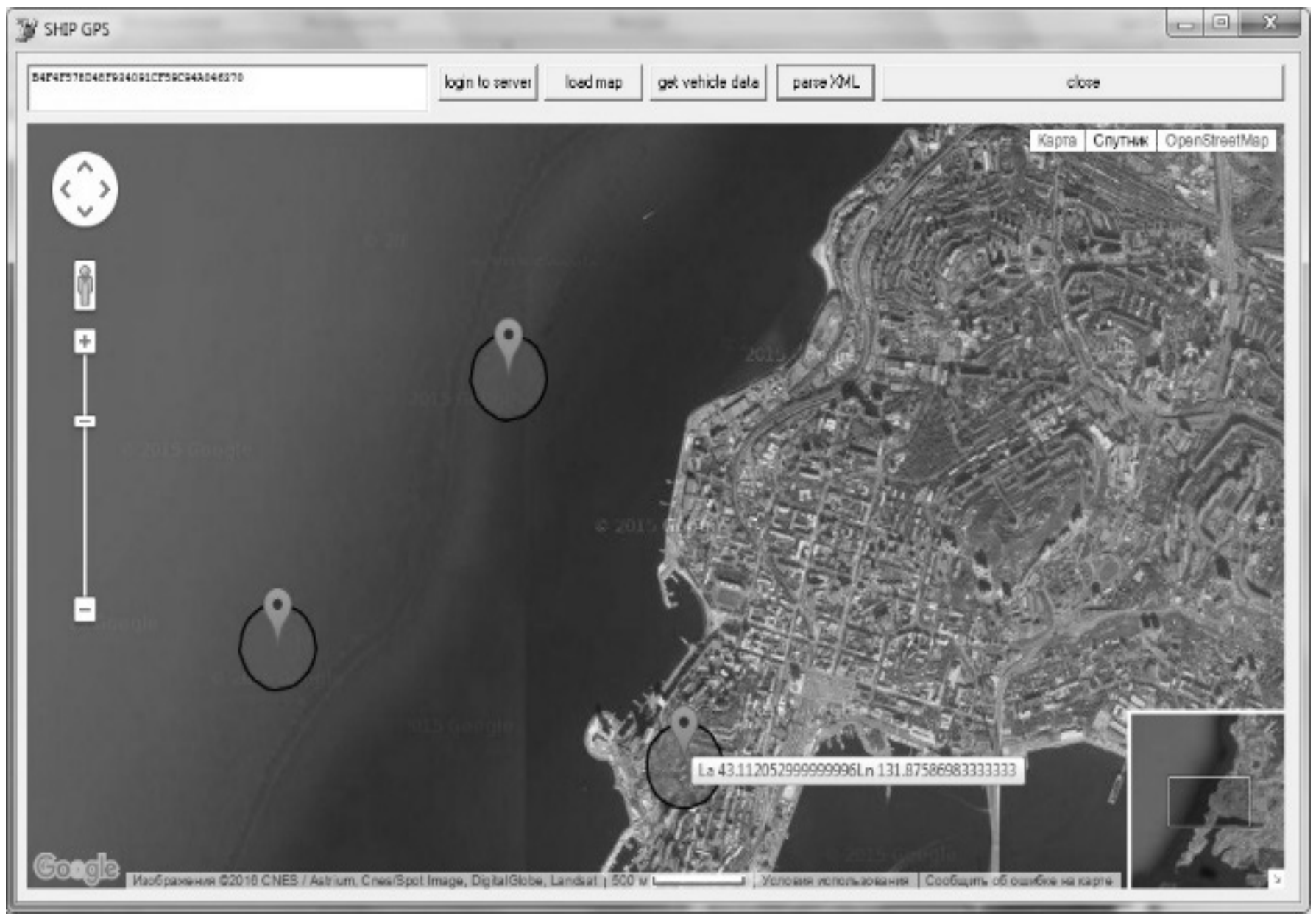

Рис. 6. Внешний вид модуля анализа и визуализации данных

этих объектов и оценивает опасность столкновения судна-оператора, идущего с известной скоростью и курсом с этими надводными объектами. Если опасность столкновения низкая, авторулевой продолжит автоматическое удержание судна на курсе, а в случае высокой опасности столкновения - оповестит лица, принимающие решения и предложит рекомендации по маневру расхождения на основе Международных правил предупреждения столкновений судов в море и выводов экспертной системы (см. рис. 2). Для реализации такого интеллектуального авторулевого ранее разработаны систеа оценки опасности столкновения и система поддержки принятия решений для судоводителя [10].

Для подтверждения действенности предложенного подхода к задаче контроля движения за надводными судами в прибрежных водах разработан прототип аппаратно-программного комплекса, в котором основная часть реализована на мобильном компьютере (ноутбук с GPS-приемником, GPRS-модемом и модулями (программным обеспечением) агрегации, визуализации и обработки данных).
Вариант реализации передающей части с использованием смартфона, как наиболее предпочтительный с точки зрения простоты использования и настройки, представлен на рисунке 3. На смартфоне используется приложение ITracklT, считывающее данные о текущем положении с встроенного GPS приемника и передающее их через сеть сотовой связи по протоколу GPRS. Приложение обеспечивает передачу данных на указанный в настройках адрес сервера с заданным интервалом.

Основная часть комплекса состоит из следующих модулей: приема данных, агрегации данных, визуализации, передачи данных во внешние системы и анализа данных. Прием данных от наблюдаемых объектов осуществляется через GPRS-модем, информация о собственном местоположении основной части комплекса считывается с GPS-приемника. Для получения GPS-данных в основной части используется приемник Vk-172 GMOUSE USB GPS. Внешний вид приемника изображен на рисунке 4.

Для агрегации данных в прототипе аппаратно-программного комплекса использовано программное обе- 
спечение Franson GPS Gate Server [11]. Оно представляет собой набор приложений для операционных систем Windows и Linux, которые позволяют принимать данные в формате National Marine Electronics Association (NMEA) [12] с различных устройств, оснащенных чипами GPS, а также агрегировать эти данные в реальном времени, визуализировать их на карте и передавать для обработки внешним приложениям. Интерфейс Franson GPS Gate Server представлен на рисунке 5.

Данные в формате NMEA содержат навигационные измерения GPS приемника - позицию, скорость и время. NMEA-сообщения состоят из последовательного набора данных, разделенных запятыми [13]. Каждое отдельное сообщение не зависит от других и является полностью «завершенным». NMEA-сообщение включает в себя заголовок, набор данных, представленных ASCII символами, и поле контрольной суммы для проверки достоверности переданной информации.

Агрегированные данные передаются в модуль анализа и визуализации данных. Интерфейс модуля представлен на рисунке 6. Этот модуль представляет собой специально разработанное для этих целей приложение, способное принимать данные через протокол обмена структурированными сообщениями (SOAP Simple Object Access Protocol) [14]. Модуль принимает данные обо всех наблюдаемых объектах, отображает их на интерактивной карте, отрисованной с использованием общедоступного программного интерфейса Google Maps.

\section{Зак^ючение}

В целях апробации предложенного метода наблюдения за надводными объектами была проведена проверка работоспособности в акватории Амурского залива Японского моря на малом боте «Галс». В ходе такой проверки была произведена передача в реальном времени информации о местоположении малого бота «Галс» с использованием смартфона с GPS-приемником в основную часть комплекса и ее обработка с последующей записью и визуализацией. Проведенный эксперимент показал, что предложенный подход можно использовать для задач наблюдения за различными малогабаритными надводными объектами в прибрежных зонах при наличии в них покрытия сетей сотовой связи. Предложенный подход к контролю за надводными объектами в прибрежной зоне предполагает использование широко распространенных мобильных устройств и бесплатного программного обеспечения, достаточно прост в реализации, точен и не требует оснащения специальным оборудованием, что позволяет положительно оценить потенциал его использования в поставленной задаче контроля надводных объектов в реальном времени.

\section{ЛИТЕРАТУРА}

1. Системы управления движением судов [Электронный ресурс]. М., 2013-2016. URL: http://seaspirit.ru/morskie-konvencii/morskoe-pravo/sistemyupravleniya-dvizheniem-sudov.html.

2. Горелик, С. Стратегические перспективы морского транспорта / С. Горелик // Транспортная стратегия — XXI век.— 2015.— № 31.— С. 14-15.

3. Скварник, И.С. Модернизация сети связи и передачи данных системы управления движением судов в заливе Петра Великого за счет средств беспроводного широкополосного доступа / И.С. Скварник // Радиоэлектроника. Проблемы и перспективы развития: Четвёртая Всероссийская молодёжная научная конференция, посвящённая дню радио, Тамбов, 06-07 мая 2019 года. - Тамбов: Издательский центр ФГБОУ В0 «Тамбовский государственный технический университет», 2019. - С. 197-199.

4. Скварник, И.С. Современные технологии в системе управления движением судов в рамках концепции е-Навигации: мировой опыт и региональные особенности (обзор) / И.С. Скварник // Вестник Инженерной школы Дальневосточного федерального университета.— 2021.— № 2(47). — C. 50-65.—D0I 10.24866/2227-6858/2021-2-6

5. Маринич А.Н. Судовая автоматическая идентификационная система / А.Н. Маринич [и др.]; под общ. ред. Ю.М. Устинова.— СПб.: Судостроение, 2004.— 179 c.

6. Сясин Д.Ю. Некоторые ограничения современных судовых идентификационных систем и методы их устранения. Сборник докладов 59-й международной молодежной научно-технической конференции «МОЛОДЕЖЬ-НАУКА-ИННОВАЦИИ», 23-25 ноября 2011 г. в 2 тт.— Владивосток: Мор. гос. ун-т, 2011.— T. 1. - c. 22-25.

7. Мельник, П.В. Концепция Е-навигации в рекомендациях имо, варианты национального исполнения и перспективы развития в судоходстве ближайшего будущего / П.В. Мельник // Вестник государственного морского университета имени адмирала Ф.Ф. Ушакова.— 2017.— № 4(21).—C. 26 -31.

8. Видеосистема наблюдения за морскими судами в реальном времени / Ш.С. Фахми, С.А. Селиверстов, Е.В. Костикова [и др.] // Морские интеллектуальные технологии.— 2021.— Т. 1.— № 2(53).—C. 98-105.—DOI 10.37220/MIT.2021.52.2.015.

9. Sedova N., Sedov V., Bazhenov R., Ledovskikh I. A Marine Autopilot With a Fuzzy Controller Computed by a Neural Network. 7th Scientific Conference on Information Technologies for Intelligent Decision Making Support (ITIDS2019), Vol. 166, 2019, p. 171-176. — Atlantis Press, 2019. https://dx.doi.org/10.2991/itids-19.2019.31.

10. Sedova N., Sedov V., Bazhenov R. Machine Learning: Neural Network Models of Sea-Craft Paths. Lecture Notes in Electrical Engineering, 729 LNEE, pp. 801-810, 2021. 
11. Комаровский, Ю.А. Сравнительный анализ точности определения координат судовым и геодезическим GPS приёмниками на малоподвижном судне / Ю.А. Комаровский // Вестник Морского государственного университета.— 2013.— № 62. - C. 75-81.

12. Попов, А.Н. Алгоритм прогнозирования для предупреждения столкновений судов в море применительно к «интеллектуальной акватории» E-NAVIGATION / А.Н. Попов, А.И. Кондратьев, Р.Э. Субанов // Эксплуатация морского транспорта. — 2018. — № 1(86). — С. 69-74.

13. Нечаев, Е.Е. Применение встраиваемых процессоров на плис для идентификации навигационных параметров / Е.Е. Нечаев // Научный вестник Московского государственного технического университета гражданской авиации. - 2010. - C. 136-142.

14. Brett McLaughlin, Илья Чекменев. Simple Object Access Protocol [Электронный ресурс], M., 2016. URL: http://www.xserver.ru/computer/langprogr/razn/104/.

○ Сясин Дмитрий Юрьевич ( dims5@mail.ru ), Седова Нелли Алексеевна ( nellyfish81@mail.ru ),

Баженов Руслан Иванович ( r-i-bazhenov@yandex.ru ), Глушков Сергей Витальевич ( glushkov@msun.ru ).

Журнал «Современная наука: актуальные проблемы теории и практики»

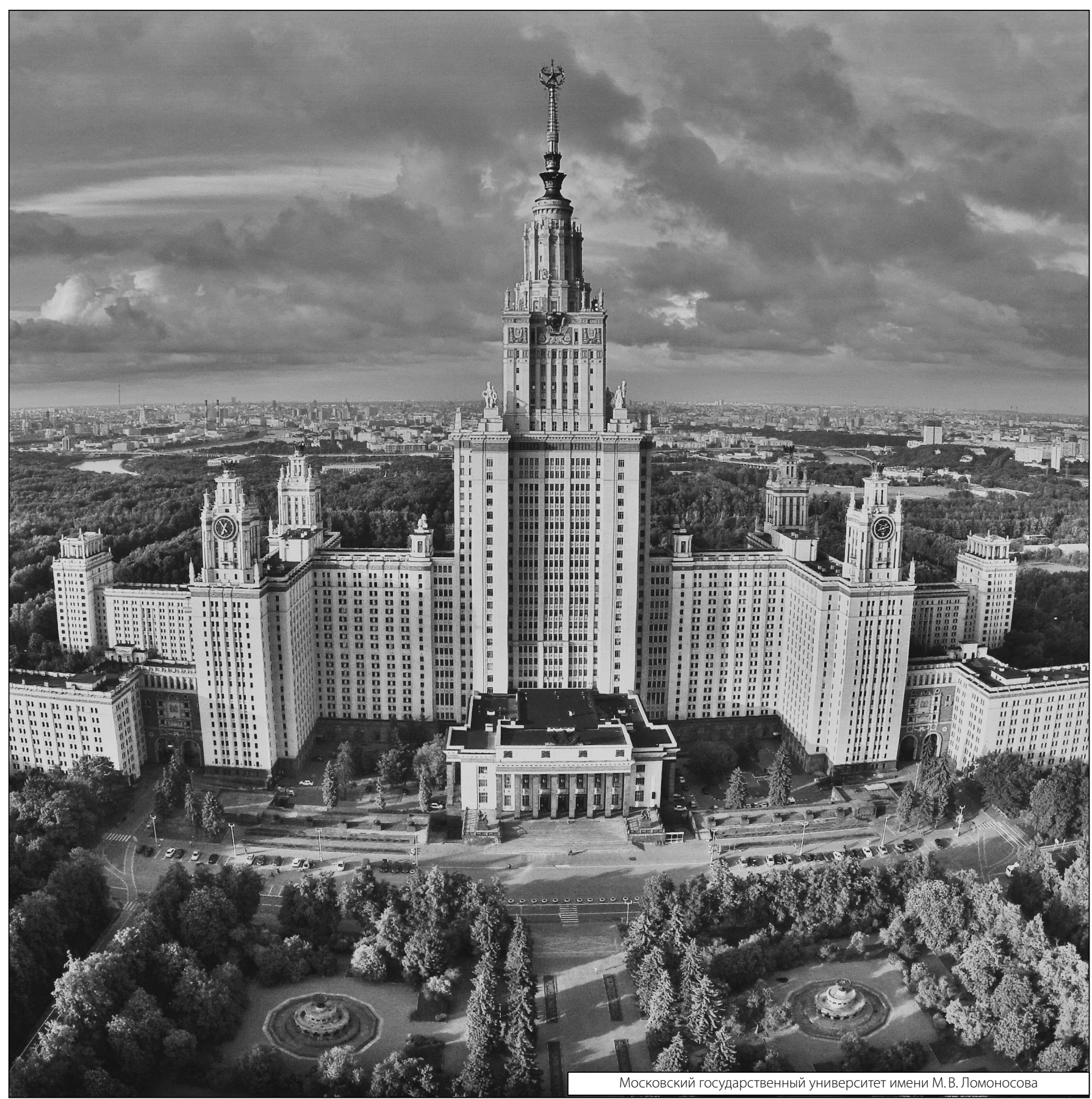

